\title{
Numerical Solution of the MHD Reynolds Equation for Squeeze-Film Lubrication between Porous and Rough Rectangular Plates
}

\author{
Ramesh B. Kudenatti, ${ }^{1}$ N. Murulidhara, ${ }^{2}$ and H. P. Patil ${ }^{3}$ \\ ${ }^{1}$ Department of Mathematics, Bangalore University, Bangalore 560 001, India \\ ${ }^{2}$ Department of Mathematics, Sri Siddhartha Institute of Technology, Tumkur 572 105, India \\ ${ }^{3}$ Department of Mathematics, Siddaganga Institute of Technology (SIT), Tumkur 572 103, India
}

Correspondence should be addressed to Ramesh B. Kudenatti; ramesh@bub.ernet.in

Received 13 December 2012; Accepted 5 January 2013

Academic Editors: J. H. Jang and S. Xiong

Copyright (C) 2013 Ramesh B. Kudenatti et al. This is an open access article distributed under the Creative Commons Attribution License, which permits unrestricted use, distribution, and reproduction in any medium, provided the original work is properly cited.

\begin{abstract}
The present theoretical study investigates the effects of surface roughness and couple-stress fluid between two rectangular plates, of which an upper rough plate has a roughness structure and the lower plate has a porous material in the presence of transverse magnetic field. The lubricant in the gap is taken to be a viscous, incompressible, and electrically conducting couple-stress fluid. This gap is separated by a film thickness $H$ which is made up of nominal smooth part and rough part. The modified Reynolds equation in the film region is derived for one-dimensional longitudinal roughness structure and solved numerically using multigrid method. The numerical results for various physical parameters are discussed in terms of pressure distribution, load capacity, and squeeze film time of the bearing surfaces. Our results show that, the pressure distribution, load capacity and squeeze film time are predominant for larger values of Hartman number and roughness parameter, and for smaller values of couple-stress parameters when compared to their corresponding classical cases.
\end{abstract}

\section{Introduction}

Magnetohydrodynamic (MHD) flow of a fluid in squeezefilm lubrication is of interest, because it prevents the unexpected variation of lubricant viscosity with temperature under severe operating conditions. The effects of magnetic field in squeeze lubrication have been encouraging because magnetic field has important applications in the industry with obvious relevance to technology-based world. The MHD lubrication in an externally pressurized thrust bearing has been investigated both theoretically and experimentally by Maki et al. [1]. Limited studies of MHD lubrication are available in the literature which includes MHD slider bearings $[2,3]$, MHD journal bearings [4, 5], and MHD squeeze film bearings [6]. Hamza [7] has shown the effects of MHD on a fluid film squeezed between two rotating surfaces. Bujurke and Kudenatti [8] have theoretically explored the effect of rough on electrically conducting fluid between two rectangular plates, in which an upper plate has a roughness structure. They modified the classical Reynolds equation to include the effects of roughness and magnetic field and solved it using a multigrid method. They showed that the effect of roughness and Hartmann number is to increase the pressure distribution and hence the load carrying capacity for increasing roughness and magnetic parameters.

Self-lubricating porous bearings have been studyied in the last few decades because of their industrial applications and machine manufacturing. These bearings have selfcontained oil reservoir and hence do not require continuous lubrication. Most porous bearings have interconnecting pores which store the lubricating fluid. When the normal load is applied, the fluid is supplied through the interconnected pores to the fluid film region to support the load, and when the load is removed from the loaded zone of the bearing, 
fluid is reabsorbed by capillary action. Since these can operate without additional lubricant for longer period, porous bearings have been used widely, where relubrication would be difficult. Thus, porous metal bearings have been used in the manufacturing of vehicles, home appliances, machines, and so forth. Because of the importance of porous bearings, $\mathrm{Wu}$ [9] studied the squeeze film effects between two rectangular plates in which both plates have a porous material. Numerous papers are available in the literature for the study of different types of porous bearings for example, journal bearings [10], slider bearings [11], thrust bearings [12], and many more. Naduvinamani et al. [13] have undertaken a detailed study of magnetic effects in rectangular plates and reported that bearing characteristics such as pressure distribution, load capacity, and squeezing time seem to increase for increasing the Hartman number.

When the gap between two mating surfaces becomes smaller, the effects of roughness become more important. In most of the applications, the smooth bearing surface would not be valid for the accurate prediction of the performance and life of the bearings. Thus, surface roughness has been studied with much interest in the recent years because all bearing surfaces are rough to some extent. Also to increase the performance of hydrodynamic lubrication in different bearings, it is important to study the influence of surface roughness. To study this, many theories have been proposed and implemented in the context of surface lubrication, the efforts were made by Christensen [14], Christensen and Tonder [15], and Chow and Cheng [16] within the framework of the stochastic theory, but their studies were restricted to two-dimensional roughness patterns. Later, Patir and Cheng [17] considered the flow between rough surfaces in which the flow is equated to an averaged flow between two smooth surfaces, and the roughness parameter is included directly in the Reynolds equation. However, this approach fails when the random roughness structures are not identical. Christensen [15] stochastic model which assumes that the probability density function for the random variable characterizing the roughness is symmetric with the mean of the random variable equal to zero. According to Christensen's model, there are two types of roughness patterns which are of special interest in the roughness theory. The Reynolds equation which is derived to incorporate roughness structure necessarily includes onedimensional longitudinal and transverse roughness structures. In one-dimensional longitudinal case, the roughness striations have the form of long narrow ridges and valleys running in the $x$-direction, whereas in one-dimensional transverse case these striations run in $y$-direction in the form of long narrows and valleys. In case of rectangular geometry, only one of the above two cases has been studied (longitudinal case in particular) because the other one can be obtained by just coordinating transformation. Because of this considerable transformation, many theories have been studied in the literature. Bujurke and Naduvinamani [18] have studied the effect of roughness on squeeze film characteristics between two rectangular plates of which the upper plate has roughness structure and the lower plate has a porous material using Christensen [15] stochastic theory. This study shows that the effect of a rough is to increase the load capacity of the bearing compared to smooth case. Litwin [19] has experimentally investigated the effect of roughness on the water-lubricated polymer bearings and showed that the effect of roughness transversal to the sliding direction of the bearing can increase hydrodynamic load capacity.

The study of squeeze film performance in the above investigations assumes that the lubricant in the fluid film region behaves essentially as a Newtonian fluid. The classical Newtonian theory does not give satisfactory results for many engineering problems. Experimental studies show that the addition of small amount of additives into a nonpolar fluid provides significant enhancement on the bearing characteristics. Since the classical Newtonian theory cannot accurately describe the rheological behaviour of lubricants blended with various additives, a number of microcontinuum theories have been proposed. Stokes [20] theory was the simplest generalization of the classical theory of fluids which allows polar effects such as the presence of couple stresses and body couples. Essentially this theory describes the peculiar behaviour of fluids containing substructure and is intended to account for particle size effects. Couple-stress fluids consist of rigid, randomly oriented particles suspended in a viscous fluid such as electrorheological fluids and synthetic fluids. Couple stresses may appear particularly in problems where thin films exist. Many authors have used this couple-stress fluid model to study the various hydrodynamic lubrication problems, for example, journal bearings [21, 22], porous circular disks [23], slider bearings [24], MHD squeeze film bearings [25], and so on.

To the authors' knowledge, so far no attempt has been made to investigate effects of roughness and non-Newtonian couple-stress fluid on hydrodynamic lubrication between two rectangular plates, of which the lower plate has a porous material and the upper one has a roughness structure, in the presence of uniform magnetic field.

The presentation of the paper proceeds as follows. In Section 2, all the basic MHD equations with appropriate boundary conditions in both fluid film and porous region are given. From governing equations, the modified onedimensional Reynolds equation in nondimensional form is derived in Section 3. Finite difference-based multigrid method for the solution of the MHD Reynolds equation is given in Section 4. Section 5 discusses the various important results such as pressure distribution, load-carrying capacity, and squeezing time and so forth. Final section summarizes the important findings and their usefulness in designing bearings.

\section{Mathematical Formulation}

Figure 1 shows a schematic diagram of the squeeze-film lubrication between two rectangular plates. The upper rough plate approaches the lower porous plate with constant velocity $-\partial H / \partial t$. The lubricant in the film region consists of flow of viscous isothermal and incompressible electrically conducting couple-stress fluid. In addition to the usual assumptions of lubrication theory, the fluid inertia is negligible except the Lorentz force, the body forces are also neglected. Under these 
assumptions, the hydrodynamic lubrication theory applicable to thin films the MHD governing equations of motion in rectangular coordinate are

$$
\begin{gathered}
\frac{\partial p}{\partial x}=\mu \frac{\partial^{2} u}{\partial y^{2}}-\eta \frac{\partial^{4} u}{\partial y^{4}}-\sigma B^{2} u, \\
\frac{\partial p}{\partial y}=0, \\
\frac{\partial p}{\partial z}=\mu \frac{\partial^{2} w}{\partial y^{2}}-\eta \frac{\partial^{4} w}{\partial y^{4}}-\sigma B^{2} w, \\
\frac{\partial u}{\partial x}+\frac{\partial v}{\partial y}+\frac{\partial w}{\partial z}=0,
\end{gathered}
$$

where $u, v$, and $w$ are the velocities of the fluid in $x$-, $y$ - and $z$-direction, respectively, $p$ is the pressure distribution in the fluid region, and $\mu$ is viscosity of the fluid, $\sigma$ is the electrical conductivity of the lubricant, $B$ is the impressed magnetic field. The introduction of $\eta$ in (1) and (3) above is responsible for polar additives in the nonpolar lubricant. Quantity $\eta / \mu$ has the dimension of length squared which characterizes the couple-stress fluid. The required boundary conditions for the velocity components are

$$
\begin{aligned}
& \text { at } y=0: \quad u=0, \quad w=0, \quad v=v^{*}, \\
& \frac{\partial^{2} u}{\partial y^{2}}=0, \quad \frac{\partial^{2} w}{\partial y^{2}}=0, \\
& \text { at } y=H: \quad u=0, \quad v=-\frac{\partial H}{\partial t}, \\
& w=0, \quad \frac{\partial^{2} u}{\partial y^{2}}=0, \quad \frac{\partial^{2} w}{\partial y^{2}}=0,
\end{aligned}
$$

where the last two conditions in (5a) and (5b) are vanishing the couple-stress fluid at the bearing surfaces, and $\partial H / \partial t$ is an approaching velocity of the upper plate to the lower plate. The thin gap is separated by thickness $H$ which is made up of two parts

$$
H=h(t)+h_{s}(x, z, \xi),
$$

where $h(t)$ is the height of the nominal smooth part of the fluid film region, and $h_{s}(x, z, \xi)$ is the part due to the surface asperities measured from the nominal level and is a randomly varying quantity of zero mean, and $\xi$ is the index parameter determining a definite roughness structure.

The equation of motion of couple-stress fluid through a porous region can be derived as follows. The modified form of Darcy's law, which governs the flow of a couple-stress fluid in the porous region and in the presence of applied magnetic field, can be derived from the Stokes [20] equation

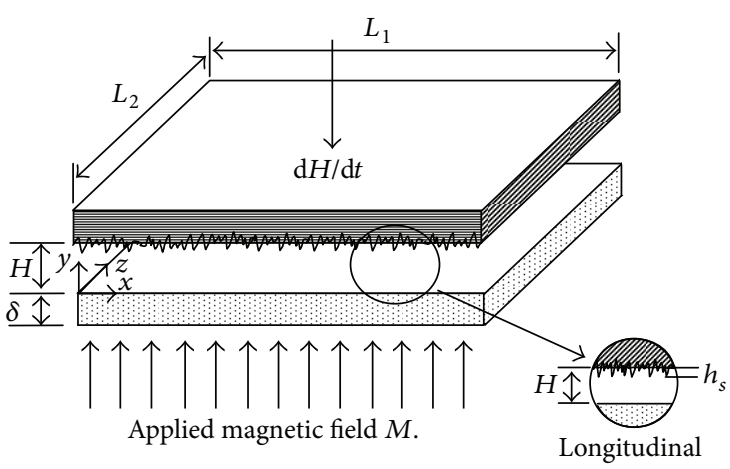

Figure 1: The physical configuration of squeeze-film lubrication between two rectangular plates in which the lower plate has a porous material and the upper plate has a rough structure under uniform magnetic field $M$. The film thickness $H$ contains height of nominal smooth part and roughness. The upper plate approaches the lower one with velocity $d H / d t$.

by statistical averages, and after simplifications, which are given by

$$
\begin{gathered}
u^{*}=-\frac{k}{\mu\left(1+\varphi M^{2}-\beta\right)} \frac{\partial P^{*}}{\partial x}, \\
v^{*}=-\frac{k}{\mu(1-\beta)} \frac{\partial P^{*}}{\partial y}, \\
w^{*}=-\frac{k}{\mu\left(1+\varphi M^{2}-\beta\right)} \frac{\partial P^{*}}{\partial z}, \\
\nabla \cdot \mathbf{q}^{*}=0,
\end{gathered}
$$

where $\mathbf{q}^{*}=\left(u^{*}, v^{*}, w^{*}\right), \beta=(\eta / \mu) / k$, and $M=\sqrt{\sigma / \mu} B h_{0}, P^{*}$ is the hydrostatic pressure in the porous region, $k$ is the permeability of porous material, $\varphi\left(=k / h_{0}^{2}\right)$ is nondimensional porosity parameter which is the ratio of the volume of voids on the porous material to the total volume, $M\left(=\sqrt{\sigma / \mu} B h_{0}\right)$ is the nondimensional magnetic (Hartmann) parameter which is the ratio of electromagnetic force to the viscous force, and $h_{0}$ is the minimum thickness. If $\beta=O(1)$; then, the polar additives present in the lubricant block the pores in the porous layer, thus it reduces the Darcy flow through the porous media. The whereas for smaller value of $\beta$, the size of the polar additives is small compared to the pore size, and therefore can easily percolate into the porous medium. When $\eta=0=\beta=M,(7 \mathrm{a}),(7 \mathrm{~b})$, and (7c) reduce to the case of Newtonian fluid flow through a porous medium in the absence of magnetic field. It readily follows from (7a), (7b), and $(7 \mathrm{c})$ and $(8)$ that the pressure $P^{*}$ in the porous region obeys the Laplace equation

$$
\frac{\partial^{2} P^{*}}{\partial x^{2}}+D \frac{\partial^{2} P^{*}}{\partial y^{2}}+\frac{\partial^{2} P^{*}}{\partial z^{2}}=0
$$

where $D=\left(1+\varphi M^{2}-\beta\right) /(1-\beta)$. Integrating (9) with respect to $y$ over the porous layer thickness $\delta$ and using the solid 


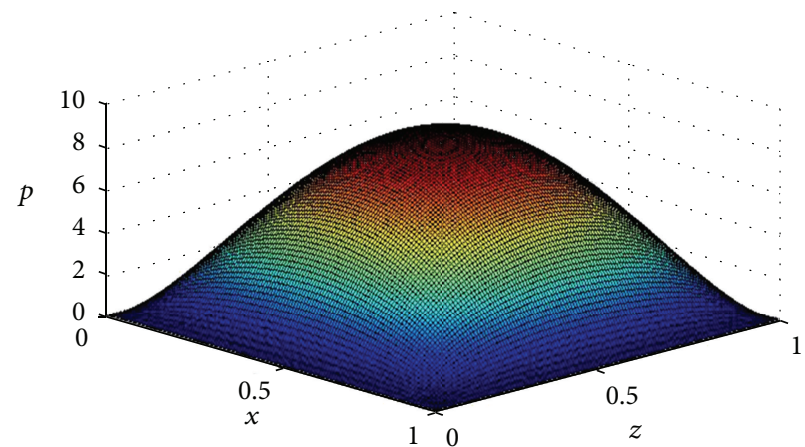

(a)

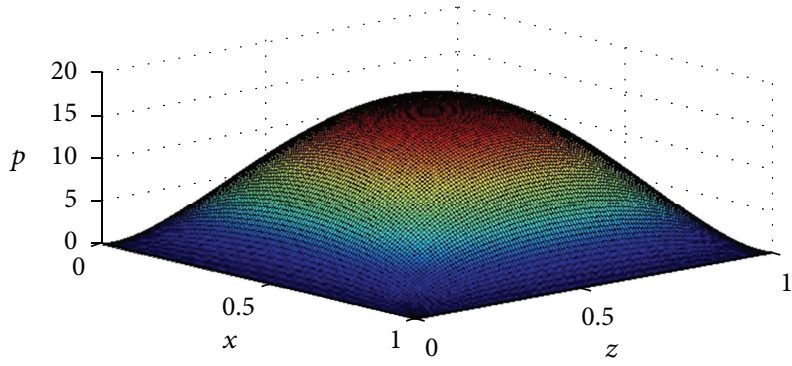

(c)

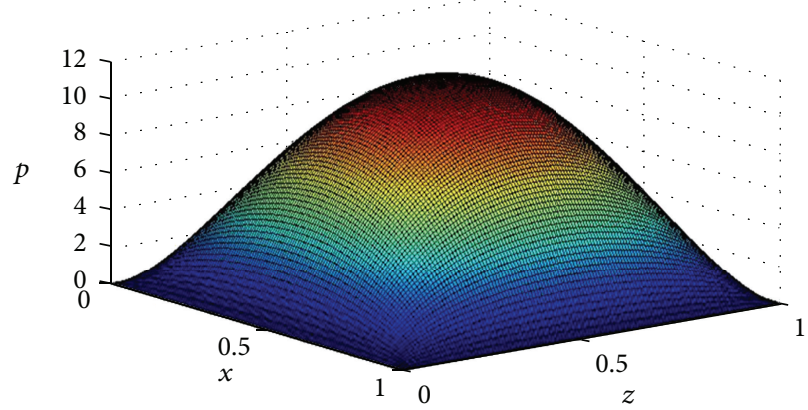

(b)

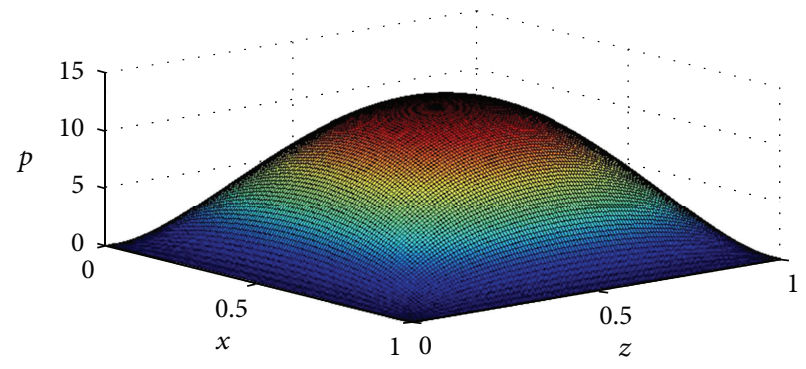

(d)

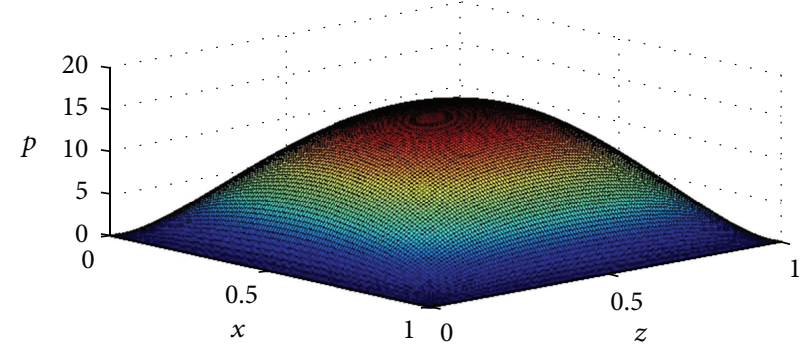

(e)

Figure 2: (a) The variation of pressure distribution $p$ for $M=1, \tau=15, C=0.2, \psi=0.0001, h=0.5$, and $\lambda=1$. (b) The variation of pressure distribution $p$ for $M=4, \tau=15, C=0.2, \psi=0.0001, h=0.5$, and $\lambda=1$. (c) The variation of pressure distribution $p$ for $M=7, \tau=15, C=$ $0.2, \psi=0.0001, h=0.5$, and $\lambda=1$. (d) The variation of pressure distribution $p$ for $M=4, \tau=10, C=0.2, \psi=0.0001, h=0.5$, and $\lambda=1$. (e) The variation of pressure distribution $p$ for $M=4, \tau=10, C=0.4, \psi=0.0001, h=0.5$, and $\lambda=1$.

backing boundary condition $\partial P^{*} / \partial y=0$ at $z=-\delta$, we get that

$$
\left.\frac{\partial P^{*}}{\partial y}\right|_{y=0}=-\frac{1}{D} \int_{-\delta}^{0}\left(\frac{\partial^{2} P^{*}}{\partial x^{2}}+\frac{\partial^{2} P^{*}}{\partial z^{2}}\right) d y,
$$

where $\delta$ is the thickness of the porous layer. Using Morgan and Cameron [26] approximation which states that the porous layer thickness is too small compared to fluid film region, and then using the pressure continuity condition $p=$ $P^{*}$ at the porous interface $y=0,(10)$ takes the form

$$
\left.\frac{\partial P^{*}}{\partial y}\right|_{y=0}=-\frac{\delta}{D}\left(\frac{\partial^{2} p}{\partial x^{2}}+\frac{\partial^{2} p}{\partial z^{2}}\right) .
$$

Thus, the governing equations which are needed to solve the problem under discussion are the momentum equations (1)(3), continuity equation (4) coupled with (11) through the boundary conditions (5a) and (5b). 


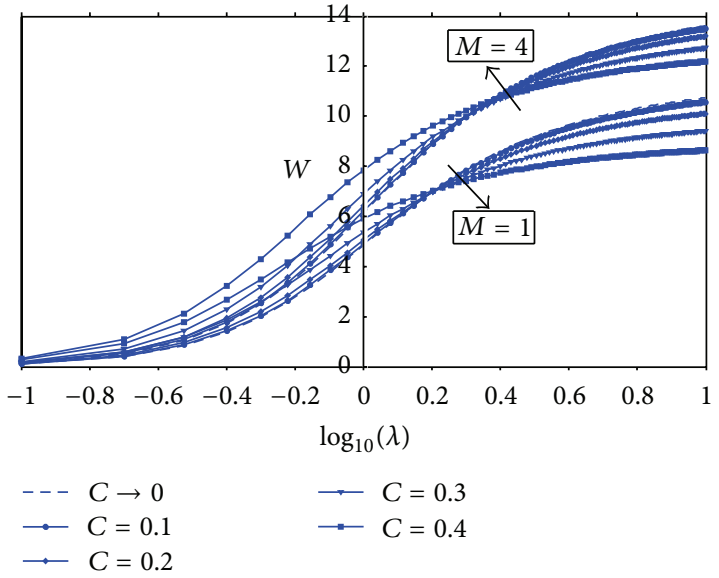

FIGURE 3: The variation of load-carrying capacity $W$ with an aspect ratio $\lambda$ for different values of roughness parameter $C$ and for two values of $M$ with $\tau=10, \psi=0.0001$, and $h=0.5$.

\section{Solution of the Problem}

Since pressure $p$ is independent of $y,(1)$ and (3) can be integrated with respect to $y$ using appropriate boundary conditions (5a) and (5b) to obtain solutions for $u$ and $w$ as a function of unknown pressure $p$. Substituting the solutions into the continuity equation (4) and then integrating it over the fluid film region, we get the modified Reynolds equation in unknown pressure as

$$
\begin{gathered}
\frac{\partial}{\partial x}\left\{F\left(H, K_{1}, K_{2}\right) \frac{\partial p}{\partial x}\right\}+\frac{\partial}{\partial z}\left\{F\left(H, K_{1}, K_{2}\right) \frac{\partial p}{\partial z}\right\} \\
=\frac{\mu M^{2}\left(K_{1}^{2}-K_{2}^{2}\right)}{h_{0}^{2}} \frac{\partial H}{\partial t}+\left.12 k \frac{\partial P^{*}}{\partial y}\right|_{y=0},
\end{gathered}
$$

where

$$
\begin{gathered}
F\left(H, K_{1}, K_{2}\right)=H\left(K_{1}^{2}-K_{2}^{2}\right)-\frac{2 K_{1}^{2}}{K_{2}} \tanh \left(\frac{K_{2} H}{2}\right) \\
+\frac{2 K_{2}^{2}}{K_{1}} \tanh \left(\frac{K_{1} H}{2}\right), \\
K_{1}=\frac{\tau}{\sqrt{2} h_{0}}\left(1+\sqrt{1-\frac{4 M^{2}}{h_{0}^{2} \tau^{2}}}\right)^{1 / 2}, \\
K_{2}=\frac{\tau}{\sqrt{2} h_{0}}\left(1-\sqrt{1-\frac{4 M^{2}}{h_{0}^{2} \tau^{2}}}\right)^{1 / 2}, \quad \tau=\sqrt{\frac{\mu}{\eta}}
\end{gathered}
$$

and $h_{0}$ is the minimum thickness. The couple-stress parameter $\tau$ has appeared for the first time and is responsible to give effect of non-Newtonian couple-stress fluid. It is expected that the effects of couple stresses are more pronounced for smaller values of $\tau$, and when $\tau$ is large, its effects are almost negligible. Substituting (11) into (12) and rearranging the terms, we get

$$
\begin{aligned}
\frac{\partial}{\partial x}\{[ & {\left.\left[F\left(H, K_{1}, K_{2}\right)+\frac{12 k \delta}{D}\right] \frac{\partial p}{\partial x}\right\} } \\
& +\frac{\partial}{\partial z}\left\{\left[F\left(H, K_{1}, K_{2}\right)+\frac{12 k \delta}{D}\right] \frac{\partial p}{\partial z}\right\} \\
= & \frac{\mu M^{2}\left(K_{1}^{2}-K_{2}^{2}\right)}{h_{0}^{2}} \frac{\partial H}{\partial t} .
\end{aligned}
$$

This modified Reynolds equation gives the pressure variation in the film region as well as in the porous region for all physical parameters. For including the effect of roughness of the bearing surfaces, we take stochastic average of the modified Reynolds equation (14) as

$$
\begin{aligned}
\frac{\partial}{\partial x}\{E & {\left.\left[\left(F\left(H, K_{1}, K_{2}\right)+\frac{12 k \delta}{D}\right) \frac{\partial p}{\partial x}\right]\right\} } \\
+ & \frac{\partial}{\partial z}\left\{E\left[\left(F\left(H, K_{1}, K_{2}\right)+\frac{12 k \delta}{D}\right) \frac{\partial p}{\partial z}\right]\right\} \\
= & \frac{\mu M^{2}\left(K_{1}^{2}-K_{2}^{2}\right)}{h_{0}^{2}} \frac{\partial E[H]}{\partial t},
\end{aligned}
$$

where $E(\cdot)$ is the expectancy operator defined by

$$
E(\cdot)=\int_{-\infty}^{\infty}(\cdot) f\left(h_{s}\right) d h_{s}
$$

and $f\left(h_{s}\right)$ is the probability density function of the stochastic variable $h_{s}$. In most of the engineering applications, roughness striations on bearing surfaces are of Gaussian type; hence, the following polynomial function which approximates the Gaussian distribution is taken in the analysis

$$
f\left(h_{s}\right)= \begin{cases}\frac{35}{32 c^{7}}\left(c^{2}-h_{s}^{2}\right)^{3}, & -c \leq h_{s} \leq c, \\ 0, & \text { otherwise, }\end{cases}
$$

where $c= \pm 3 \sigma, \sigma$ being the standard deviation.

As discussed earlier in Section 1, we restrict our study to one-dimensional longitudinal roughness. Therefore, (15) takes the form (following Christensen, for one-dimensional longitudinal roughness, we have $E\left(F\left(H, K_{1}, K_{2}\right) \partial p / \partial x\right)$ $=E\left(F\left(H, K_{1}, K_{2}\right)\right) \partial E(p) / \partial x$ and $E\left(F\left(H, K_{1}, K_{2}\right) \partial p / \partial z\right)=$ $\left.\left(1 / E\left(1 / F\left(H, K_{1}, K_{2}\right)\right)\right)(\partial E(p) / \partial z)\right)$

$$
\begin{aligned}
\frac{\partial}{\partial x}\{( & \left.\left.E\left(F\left(H, K_{1}, K_{2}\right)\right)+\frac{12 k \delta}{D}\right) \frac{\partial E(p)}{\partial x}\right\} \\
& +\frac{\partial}{\partial z}\left\{\left(\frac{1}{E\left(1 / F\left(H, K_{1}, K_{2}\right)\right)}+\frac{12 k \delta}{D}\right) \frac{\partial E(p)}{\partial z}\right\} \\
= & \frac{\mu M^{2}\left(K_{1}^{2}-K_{2}^{2}\right)}{h_{0}^{2}} \frac{\partial h}{\partial t} .
\end{aligned}
$$




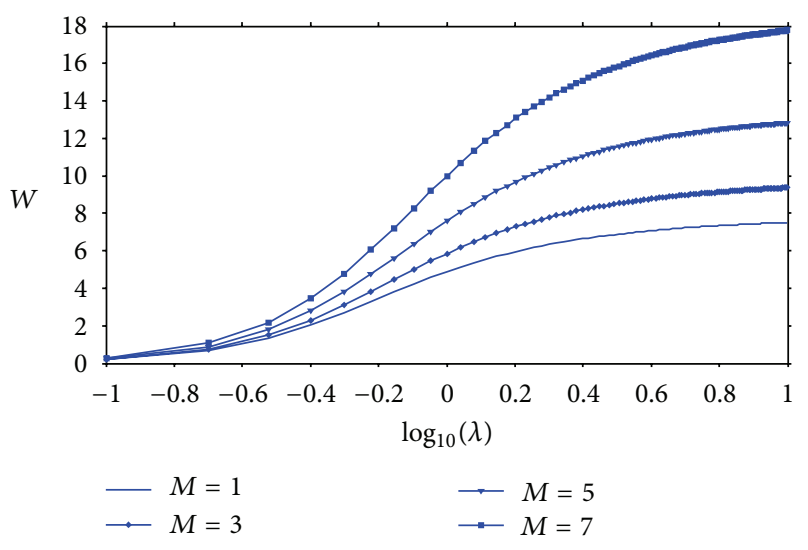

FIgURE 4: The variation of load carrying-capacity $W$ with an aspect ratio $\lambda$ for different values of Hartmann number $M$ with $C=0.2, \tau=$ $15, \psi=0.0001$, and $h=0.5$.

In order to solve the modified Reynolds equation for the pressure, the following boundary conditions are used:

$$
\begin{aligned}
E(p(0, z)) & =E\left(p\left(L_{1}, z\right)\right)=E(p(x, 0)) \\
& =E\left(p\left(x, L_{2}\right)\right)=0,
\end{aligned}
$$

where $L_{1}$ and $L_{2}$ are the dimensions of surfaces along $x$ - and $z$-directions, respectively. There is no variation of the pressure distribution outside the fluid film region. Introducing the following suitable nondimensional parameters and variables

$$
\begin{gathered}
\bar{x}=\frac{x}{L_{1}}, \quad \bar{z}=\frac{z}{L_{2}}, \quad \bar{H}=\frac{H}{h_{0}}, \\
\bar{\tau}=\tau h_{0}, \quad \psi=\frac{k \delta}{h_{0}^{3}}, \quad C=\frac{c}{h_{0}}, \\
p=-\frac{h_{0}^{3} E(p)}{L_{1}^{2} \mu \partial H / \partial t}, \quad \lambda=\frac{L_{2}}{L_{1}}
\end{gathered}
$$

into (17) and (18), we get the nondimensional Reynolds equation after retaining the original notations

$$
\begin{aligned}
\frac{\partial}{\partial x}\{ & \left.\left(E\left(F\left(H, K_{1}, K_{2}\right)\right)+\frac{12 \psi}{D}\right) \frac{\partial p}{\partial x}\right\} \\
& +\frac{1}{\lambda^{2}} \frac{\partial}{\partial z}\left\{\left(\frac{1}{E\left(1 / F\left(H, K_{1}, K_{2}\right)\right)}+\frac{12 \psi}{D}\right) \frac{\partial p}{\partial z}\right\} \\
= & M^{2}\left(K_{1}^{2}-K_{2}^{2}\right),
\end{aligned}
$$

Where

$$
\begin{gathered}
E\left(F\left(H, K_{1}, K_{2}\right)\right)=\frac{35}{32 C^{7}} \int_{-C}^{C} F\left(H, K_{1}, K_{2}\right)\left(C^{2}-h_{s}^{2}\right)^{3} d h_{s}, \\
E\left(\frac{1}{F\left(H, K_{1}, K_{2}\right)}\right) \\
=\frac{35}{32 C^{7}} \int_{-C}^{C} \frac{1}{F\left(H, K_{1}, K_{2}\right)}\left(C^{2}-h_{s}^{2}\right)^{3} d h_{s}, \\
K_{1}=\frac{\tau}{\sqrt{2}}\left(1+\sqrt{1-\frac{4 M^{2}}{\tau^{2}}}\right)^{1 / 2}, \\
K_{2}=\frac{\tau}{\sqrt{2}}\left(1-\sqrt{1-\frac{4 M^{2}}{\tau^{2}}}\right)^{1 / 2},
\end{gathered}
$$

and the boundary conditions as

$$
p(0, z)=p(1, z)=p(x, 0)=p(x, 1)=0,
$$

where $C$ is the nondimensional roughness parameter, $\psi$ is the nondimensional permeability parameter and $\lambda$ is an aspect ratio.

Thus, the modified MHD Reynolds equation (20) for longitudinal roughness structure subjected to the boundary conditions (22) is solved and simulated for pressure distribution in the fluid region for all parameters involved. Solution procedure includes the numerical solution of the both Reynolds equation and integral expressions in (21a) and (21b). For $\tau \rightarrow \infty$ and $\psi=0$, the present investigation reduces to that of the Newtonian and nonporous material studied by Bujurke and Kudenatti [8].

\section{Multigrid Method}

The modified Reynolds equation (20) is not amenable to solve analytically, because of the expressions (21a) and (21b) which are not integrable in closed form; hence, we solve it numerically using finite difference-based multigrid method. The expressions (21a) and (21b) have been numerically integrated using Simpson's 1/3rd rule. In the discretization of the Reynolds equation, standard second-order finite difference schemes are used. The field of solution is divided into 129 grids in both $x$ and $z$-directions. As it is difficult to solve these many numbers of equations using available classical methods, we resort to the convergent accelerator multigrid method for the solution of the discretized Reynolds equation. The method provides us with a simple way to compute the pressure distribution. Two grid differencing levels have been employed to approximate the Reynolds equation for good accuracy requirements. Full numerical results using $129 \times$ 129 grid level have been compared with those obtained with $257 \times 257$ grid level, the pressure distribution between two are graphically indistinguishable; thus, former grid level is adopted for further computation. The convergent solution for the pressure is obtained when the difference between 


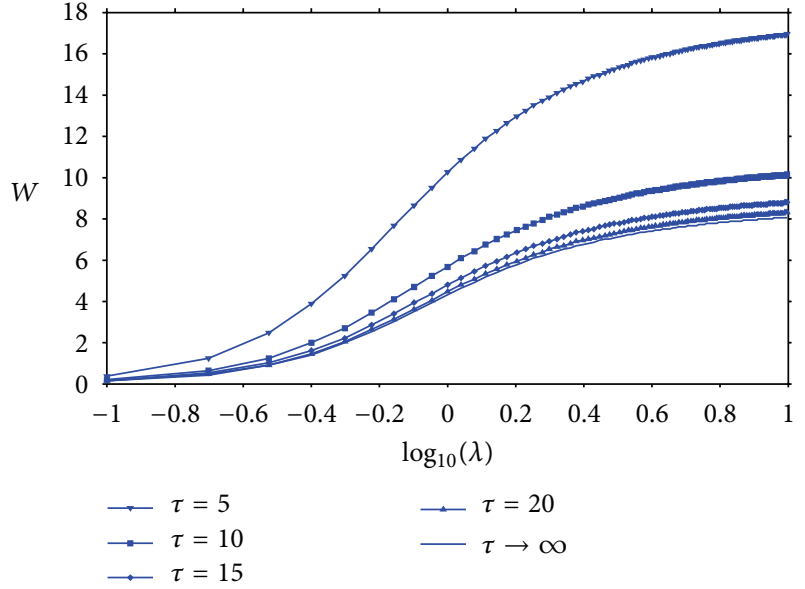

FIGURE 5: The variation of load-carrying capacity $W$ with an aspect ratio $\lambda$ for different values of couple-stress parameter $\tau$ with $M=4$, $C=0.2, \psi=0.0001$, and $h=0.5$.

pressures at two consecutive finest levels is $10^{-6}$. A detailed procedure for the solution of the Reynolds equation using multigrid method; see Bujurke and Kudenatti [27].

\section{Results and Discussion}

The behavior of bearing characteristics such as pressure distribution, load capacity, and squeeze film time can be explained by the numerical solution of the modified Reynolds equation. All required characteristic features of squeeze film bearings such as the pressure distribution, load-carrying capacity, and squeezing time. have been obtained as functions of dimensionless Hartmann number $M$, couple-stress fluid parameter $\tau$, roughness parameter $C$, aspect ratio $\lambda$, and permeability parameter $\psi$. From the Stokes [20] microcontinuum theory, a new material constant $\eta$ is responsible for couple stresses property. The parameter $\tau$ thus arises due to the presence of the polar additives in the Newtonian lubricant; hence, it is expected that effects of couple-stress fluid are more pronounced when $\tau$ is small. Also couplestress fluid provides the mechanism of the interaction of the lubricant with the bearing surfaces. The effect of roughness on the bearing surfaces can be seen by varying the parameter $C$. The case, $C \rightarrow 0$, the analysis reduces to that of perfectly smooth bearing surfaces. It is expected that for smaller values of $C$, the bearing characteristics are also not likely prominent. The various figures plotted here also reveal a comparison between smooth and roughness of the bearing surfaces, magnetic and nonmagnetic fieldness, couple stress and Newtonian lubricants, and porous and nonporous of material. For the calculation purpose, we choose $\varphi=0.6$ and $\beta=0.3$.

5.1. Nondimensional Pressure Distribution. We plot the variation of distribution of pressure as a function of rectangular coordinates $x$ and $z$ for different values of Hartmann number shown in the Figures 2(a)-2(c). These figures show the effect of magnetic field in terms of pressure distribution when other parameters are held fixed. It is observed that the pressure distribution increases for increasing values of $M$ compared to nonmagnetic field $(M=0)$. An increase in the values of $M$ makes lubricant to acquire more magnetization which in turn interacts with the magnetic field imposed. Also applied magnetic field normal to the flow reduces the velocity of the fluid in the film region which retains substantial amount of fluid. This fluid generates the pressure distribution. Also as in Figure 2(a), keeping $M=4$ fixed and varying the roughness parameter from $C=0.2$ to $C=0.5$ and decreasing the couplestress parameter from $\tau=10$ to $\tau=5$, the variations of pressure are more pronounced and are shown in Figures $2(\mathrm{~d})$ and 2(e), respectively. We note from these figures that roughness and non-Newtonian fluid promote the pressure distribution in the fluid region compared to their classical cases. This is because as the couple-stress fluid offers more resistance to the moving fluid, the larger amount of fluid would remain in the region. On the other hand, the roughness asperities present on the bearing surface also reduce the velocity of the fluid. Furthermore, the amount of leakage of lubricant along the sidewise direction is again reduced by the presence of the surface asperities. Altogether, large amount of fluid is collected in the region which generates large pressure distribution. These differences can be seen clearly from these figures. Thus, the magnetic field, roughness, and non-Newtonian fluid are favorable factors for increasing pressure distribution.

5.2. Nondimensional Load-Carrying Capacity. To understand effects of all physical quantities, it is important to plot the variation of load-carrying capacity with other physical quantities. The nondimensional load carrying capacity can be obtained by integrating the pressure distribution across the fluid region. The nondimensional load-carrying capacity $W$ of the bearing surface per unit area is given by

$$
\begin{aligned}
W & =-\frac{E(W) h_{0}^{3}}{\mu L_{1}^{2} L_{2}^{2} \partial h / \partial t}=\iint_{0}^{1} p(x, z) d x d z \\
& =(\Delta x)^{2} \sum_{m=1}^{N} \sum_{n}^{N} p_{m, n},
\end{aligned}
$$

where $\Delta x=\Delta z$ is the grid size used the finite difference scheme. Results obtained for load-carrying capacity are shown in Figures 3-6 for various parameters. The variation of load capacity $W$ with aspect ratio $\log _{10}(\lambda)$ for different values of roughness parameters and for two values of Hartmann number $M$ where all other parameters held constant is shown in Figure 3. It is interesting to note that there exists a critical aspect ratio $\lambda_{C}(M)$, where the effects of roughness will vanish. At a critical value $\lambda_{C}$ (in the present study, $\lambda_{C}=$ 1.2503 for $M=1$ and $\lambda_{C}=1.4761$ for $M=4$ ), the effect of roughness is to increase $W$ for $\lambda<\lambda_{C}$, and the trend is reversed for the other case. The same trend has also been observed for other magnetic field. Also it is observed that the magnetic field increases the load-carrying capacity. 


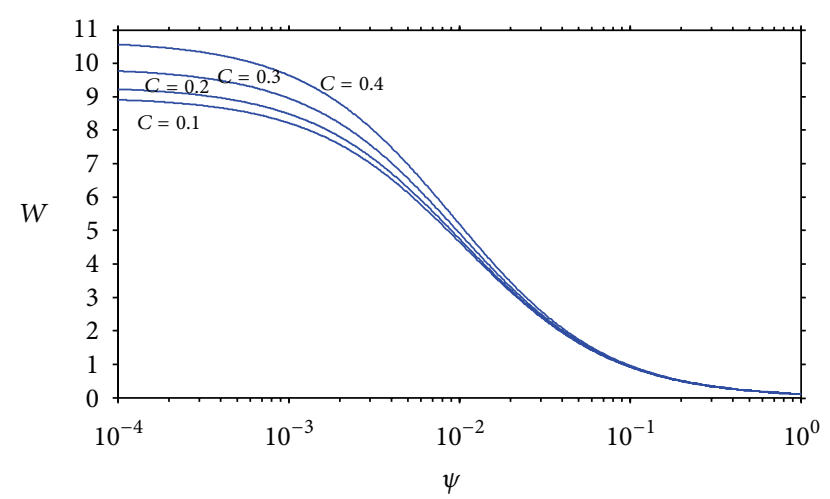

FIgURE 6: The variation of load-carrying capacity $W$ with a permeability parameter $\psi$ for different values of roughness parameter $C$ with $M=2, \tau=5$, and $h=0.5$.

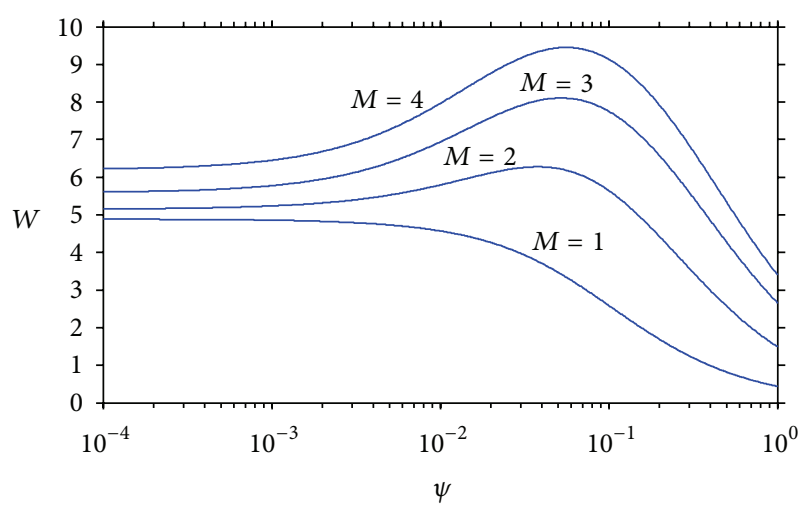

FIGURE 7: The variation of load-carrying capacity $W$ with a permeability parameter $\psi$ for different values of Hartmann number $M$ with $C=0.1, \tau=10$, and $h=0.5$.

In Figure 4, we plot the variation of load capacity $W$ with an aspect ratio $\log _{10}(\lambda)$ for different values of magnetic number $M$ when all other parameters held constant. The doted curve represents the classical case, that is, the nonmagnetic field. It is clearly observed from this figure that the load capacity increases for increasing magnetic number compared to $M=0$. As explained in previous section, for increasing values of $M$, pressure distribution increases in the fluid region, hence, load capacity also increases. The variation of load capacity $W$ with an aspect ratio $\log _{10}(\lambda)$ for different values of couple-stress parameter $\tau$ keeping other parameters constant is shown in Figure 5. The curve without symbol represents the Newtonian case (i.e., $\tau \rightarrow \infty$ ). It is noted from the figure in contrast to the earlier discussion the load capacity decreases for increasing values of $\tau$. Furthermore, as aspect ratio $\lambda$ increases from 0.1 to 10 , the load capacity also increases, and this typical trend is observed for all values of $M$ and $\tau$.

The variations of load capacity with that of the permeability parameter $\psi$ for different values of roughness parameter are shown in Figure 6. It is interesting to note that the effect of permeability parameter is to decrease the mean load capacity for all values of $C$, whereas for increasing roughness parameter $C$, the mean load capacity also increases. The obvious reason for this to happen is that larger value of $\psi$ leads to more number of voids on the porous facing, allowing the fluid to percolate into a porous region. Thus, pores on the porous facing become the main path for fluid to flow. This decreases the quantity of fluid in the film region, hence, pressure generation also decreases, so with load capacity. However, this significant lost in load capacity could be compensated by appropriate values of roughness parameters so that lost can be regained. This is evident from Figure 7. It is interesting to note that except $M=1.0$, for all other values of $M$, the load capacity increases gradually for the first few values of $\psi$ and for some values of $\psi$, it further increases before starts to decrease for increasing $\psi$. However, this trend is not observed for $M=1$. The effect of porous is to decrease the load capacity for all values of $C, \tau$, and $M$.

5.3. Nondimensional Squeeze Time-Height Relation. Another most important bearing characteristic is the squeeze film time, that is, the time taken by the upper plate to reach a film thickness $h$ that can be determined in a nondimensional form as

$$
T=\frac{h_{0}^{2} E(W) t}{\mu L_{1}^{2} L_{2}^{2}}=\int_{0}^{1} W d h=\iiint_{0}^{1} p(x, z) d x d z d h .
$$

Various results for squeeze film time $T$ are obtained as function of Hartmann number $M$ and couple-stress parameter $\tau$ that have been shown in Figures 8 and 9. Figure 8 shows the variation of squeeze film time $T$ as a function of squeeze film height for different values of Hartmann number $M$ when other physical parameters held constant. It is observed that the squeezing time decreases as the film height increases. Also it is worth to mention that the effect of the magnetic field is clearly seen. As magnetic parameter increases the squeeze film time also increases. The applied magnetic field strongly opposes the fluid flow in the film region which results large number of fluid would remain in the film region. Thus, the squeezing time increases significantly disclosing the fact that the magnetic field offers the delayed squeeze film of the upper plate which reduces the coefficient of friction. The similar results also have been observed from Figure 9, wherein we give the variation of film height as a function of couplestress parameter $\tau$ keeping other parameters constant. The dashed curve represents the Newtonian fluid $(\tau \rightarrow \infty)$. The effect of couple-stress parameter is to increase the squeeze time compared to the Newtonian case. While discussing the effects of $\tau$ for load capacity (Figure 5 ) in the previous section, we claim that the couple-stress fluid offers more delayed squeezing time compared to Newtonian case. We expect the similar results for roughness parameters that also increase the squeeze time which is not shown here for brevity. Thus, we conclude that the applied magnetic field together with couple-stress fluid promote the squeezing time of the upper 


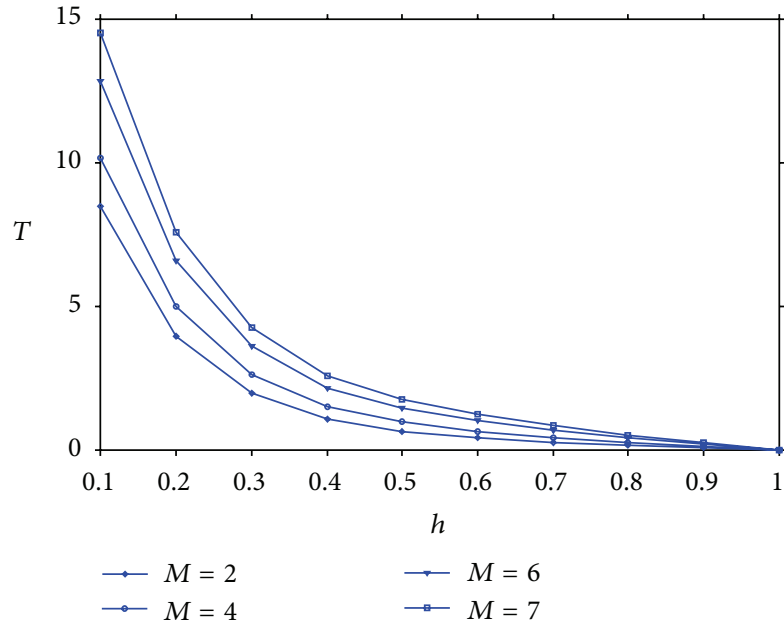

FIGURE 8: The variation of nondimensional squeeze film time $T$ with film thickness $h$ for different values of magnetic parameter $M$ with $C=0.4, \psi=0.0001$, and $\tau=15$.

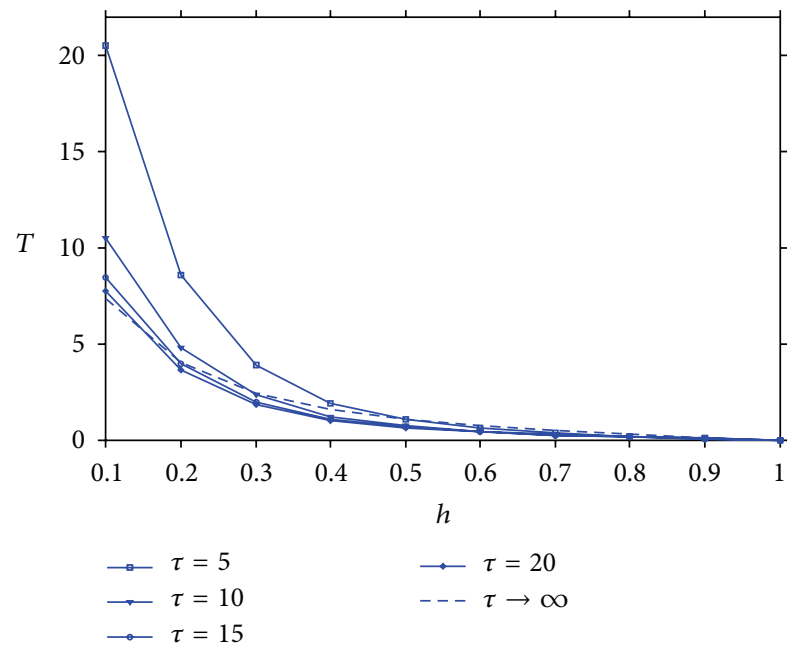

FIGURE 9: The variation of nondimensional squeeze film time $T$ with film thickness $h$ for different values of couple-stress parameter $\tau$ with $C=0.4, \psi=0.0001$, and $M=2$.

plate and help to reduce the coefficient of friction and rate of wear of the plates.

\section{Conclusions}

The application of finite difference-based multigrid method for the solution of modified Reynolds equation to investigate the combined effects of surface roughness, magnetic field, couple-stress fluid, permeability, and so forth has confirmed important features of squeeze-film lubrication between two finite rectangular plates. The modified MHD Reynolds equation is derived on the basis of Christensen stochastic theory and Stokes theory for couple-stress fluid. It is observed that the pressure distribution, load-carrying capacity, and squeeze time increase for increasing values of roughness parameter and Hartmann number and smaller values of couple-stress parameter, whereas these are found to decrease for increasing permeability compared to their corresponding classical cases. These physical quantities have considerable influence on the performance of the bearings. It is expected that these findings help the design engineers to choose an appropriate roughness structure, magnetic field, and lubricant additives in order to enhance the normal functioning of the bearing life.

\section{References}

[1] E. R. Maki, D. C. Kuzma, and R. L. Donnelly, "Magnetohydrodynamic lubrication flow between parallel plates," Journal of Fluid Mechanics, vol. 26, no. 3, pp. 537-543, 1966.

[2] M. I. Anwar and C. M. Rodkiewicz, "Nonuniform magnetic field effects in MHD slider bearing," Journal of Lubrication Technology, vol. 94, no. 1, pp. 101-105, 1972.

[3] N. C. Das, "Study of optimum load capacity of slider bearings lubricated with power law fluids," Tribology International, vol. 32, no. 8, pp. 435-441, 1999.

[4] S. Kamiyama, "Magnetohydrodynamic journal bearing (report 1)," Journal of Lubrication Technology, vol. 91, no. 3, pp. 380-389, 1969.

[5] M. Malik and D. V. Singh, "Analysis of finite magnetohydrodynamic journal bearings," Wear, vol. 64, no. 2, pp. 273-280, 1980.

[6] J. B. Shukla, "Hydromagnetic theory for squeeze films," Journal of Basic Engineering, vol. 87, no. 1, pp. 142-144, 1965.

[7] E. A. Hamza, "Magnetohydrodynamic effects on a fluid film squeezed between two rotating surfaces," Journal of Physics D, vol. 24 , no. 4 , pp. 547-554, 1991.

[8] N. M. Bujurke and R. B. Kudenatti, "MHD lubrication flow between rough rectangular plates," Fluid Dynamics Research, vol. 39, no. 4, pp. 334-345, 2007.

[9] H. Wu, "Analysis of the squeeze film between porous rectangular plates," Journal of Lubrication Technology, vol. 94, no. 1, pp. 64-68, 1972.

[10] J. Prakash and S. K. Vij, "Load capacity and time-height relations for squeeze films between porous plates," Wear, vol. 24, no. 3, pp. 309-322, 1973.

[11] U. Srinivasan, "The analysis of a double-layered porous slider bearing," Wear, vol. 42, no. 2, pp. 205-215, 1977.

[12] R. S. Gupta and V. K. Kapur, "Centrifugal effects in hydrostatic porous thrust bearings," Journal of Lubrication Technology, vol. 101, no. 3, pp. 381-392, 1979.

[13] N. B. Naduvinamani, S. T. Fathima, and S. Jamal, "Effect of roughness on hydromagnetic squeeze films between porous rectangular plates," Tribology International, vol. 43, no. 11, pp. 2145-2151, 2010.

[14] H. Christensen, "Stochastic models for hydrodynamic lubrication of rough surfaces," Proceedings of the Institution of Mechanical Engineers, vol. 184, pp. 1022-1033, 1969.

[15] H. Christensen and K. Tønder, "Tribology of rough surfaces, parametric study and comparison of lubrication models," SINTEF Research Report No. 22/69-18, 1969.

[16] L. S. H. Chow and H. S. Cheng, "The effect of surface roughness on the average film thickness between lubricated rollers," Journal of Lubrication Technology, vol. 98, no. 1, pp. 117-124, 1976. 
[17] N. Patir and H. S. Cheng, "An average flow model for determining effects of three-dimensional roughness on partial hydrodynamic lubrication," Journal of Lubrication Technology, vol. 100, no. 1, pp. 12-17, 1978.

[18] N. M. Bujurke and N. B. Naduvinamani, "A note on squeeze film between rough anisotropic porous rectangular plates," Wear, vol. 217, no. 2, pp. 225-230, 1998.

[19] W. Litwin, "Influence of surface roughness topography on properties of water-lubricated polymer bearings: experimental research," Tribology Transactions, vol. 54, no. 3, pp. 351-361, 2011.

[20] V. K. Stokes, "Couple stresses in fluids," Physics of Fluids, vol. 9, no. 9, pp. 1709-1715, 1966.

[21] H. L. Chiang, C. H. Hsu, and J. R. Lin, "Lubrication performance of finite journal bearings considering effects of couple stresses and surface roughness," Tribology International, vol. 37, no. 4, pp. 297-307, 2004.

[22] N. B. Naduvinamani and S. B. Patil, "Combined effects of surface roughness and couple stresses on static and dynamic behaviour of squeeze film lubrication of porous journal bearings," Tribology-Materials, Surfaces and Interfaces, vol. 1, no. 3, pp. 145-153, 2007.

[23] N. M. Bujurke, D. P. Basti, and R. B. Kudenatti, "Surface roughness effects on squeeze film behavior in porous circular disks with couple stress fluid," Transport in Porous Media, vol. 71, no. 2, pp. 185-197, 2008.

[24] S. F. Alyaqout and A. A. Elsharkawy, "Optimal film shape for two-dimensional slider bearings lubricated with couple stress fluids," Tribology International, vol. 44, no. 3, pp. 336-342, 2011.

[25] R. B. Kudenatti, D. P. Basti, and N. M. Bujurke, "Numerical solution of the MHD Reynolds equation for squeeze film lubrication between two parallel surfaces," Applied Mathematics and Computation, vol. 218, no. 18, pp. 9372-9382, 2012.

[26] V. T. Morgan and A. Cameron, "The mechanism of lubrication on porous metal bearings," in Proceedings of the Conference on Lubrication and Wear, vol. 89, pp. 151-157, Institute of Mechanical Engineers, London, UK, 1957.

[27] N. M. Bujurke and R. B. Kudenatti, "Multigrid solution of modified reynolds equation incorporating poroelasticity and couple stresses," Journal of Porous Media, vol. 10, no. 2, pp. 125136, 2007. 

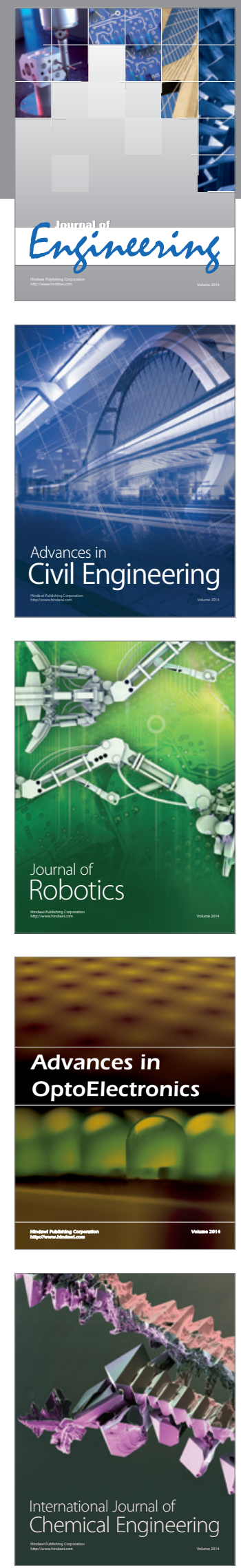

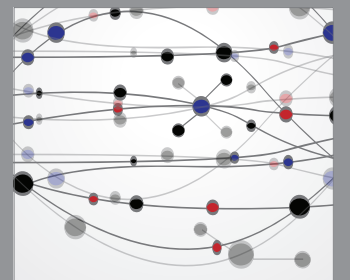

The Scientific World Journal
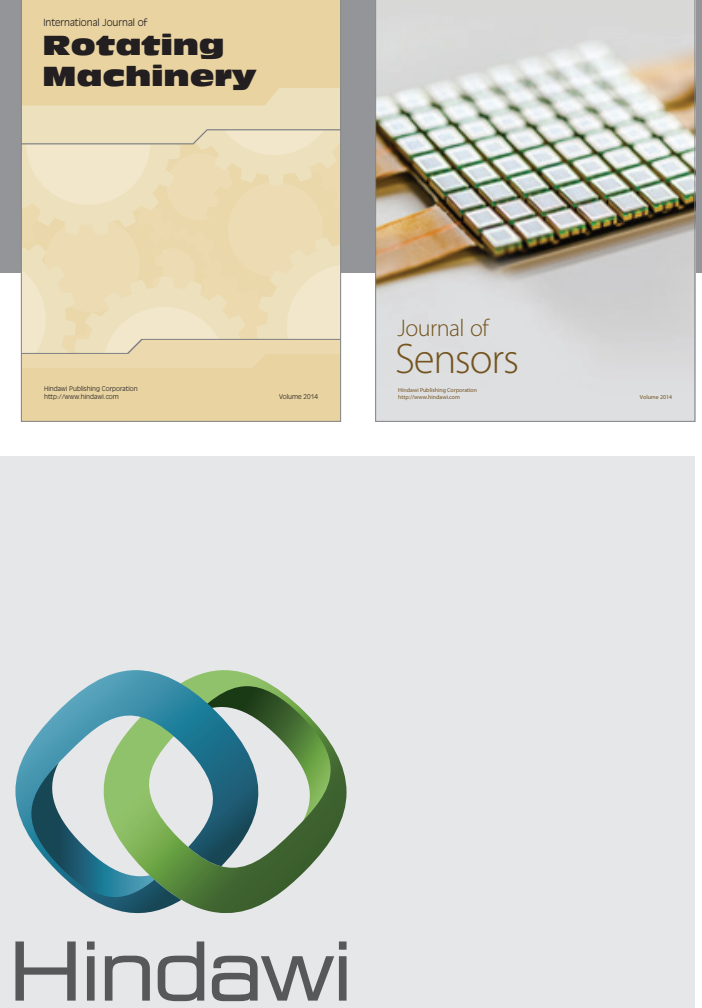

Submit your manuscripts at http://www.hindawi.com
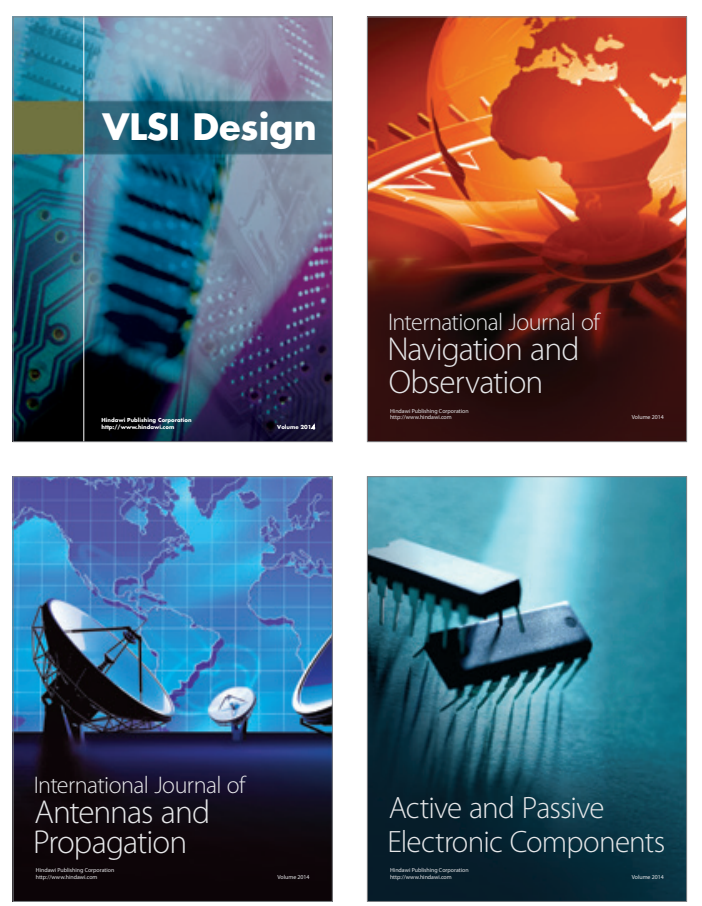
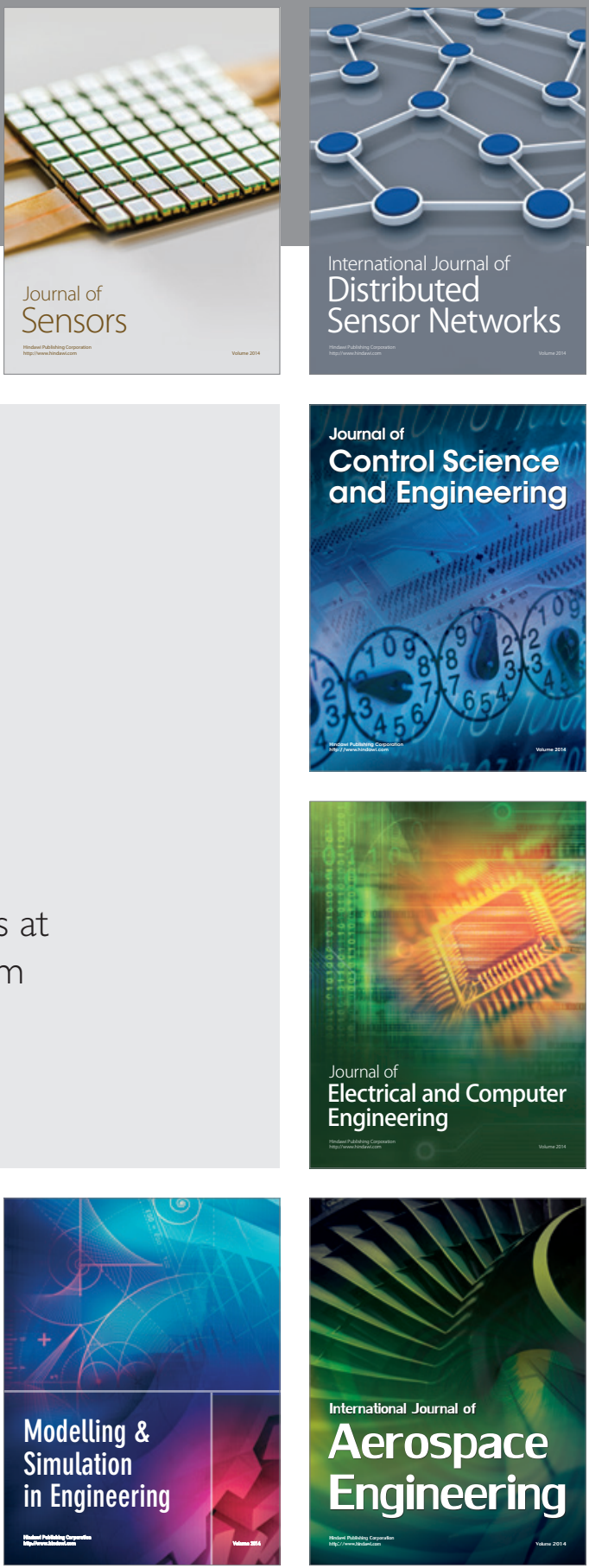

Journal of

Control Science

and Engineering
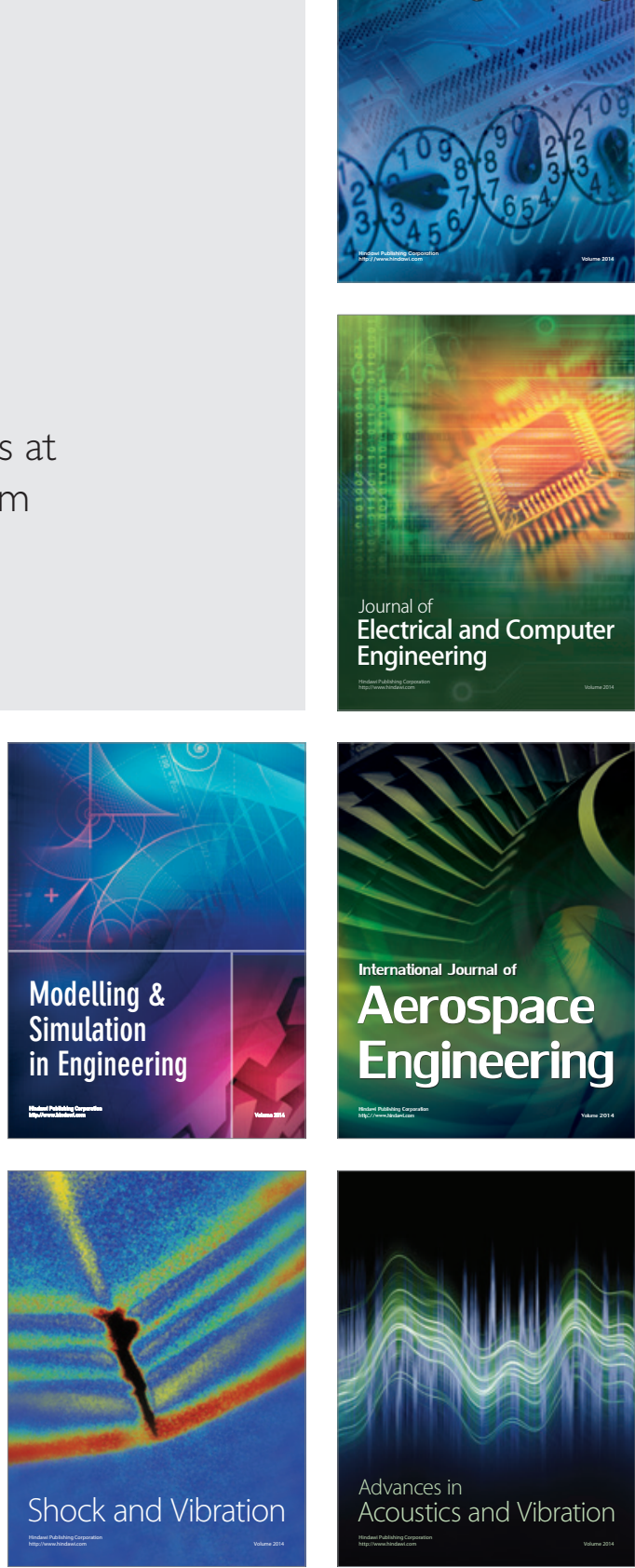